\title{
Commentary to an Article: "Molecular Mechanisms Underlying Oxytocin-Induced Cardiomyocyte Protection From Simulated Ischemia-Reperfusion"
}

\author{
Marek Jankowski ${ }^{1,2 *}$, Tom L. Broderick ${ }^{3}$, Jolanta Gutkowska',2\# \\ Cardiovascular Biochemistry Laboratory, CRCHUM (7-134), Tour Viger, 900 St-Denis St, Montreal, Canada H2X 0A9, USA \\ ${ }^{2}$ Department of Medicine, University of Montreal, Quebec, Canada, USA \\ ${ }^{3}$ Department of Physiology, Laboratory of Diabetes and Exercise Metabolism, Midwestern University, Glendale, AZ, USA
}

Article Info

\section{Article Notes}

Received: October 9, 2018

Accepted: November 1, 2018

\section{${ }^{*}$ Correspondence:}

*Dr. Marek Jankowski, Cardiovascular Biochemistry Laboratory, CRCHUM (7-134), Tour Viger, 900 St-Denis

St., Montreal, Quebec, Canada H2X OA9, USA; E-mail: jankowsm46@gmail.com.

"Dr. Jolanta Gutkowska, Cardiovascular Biochemistry Laboratory, CRCHUM (7-134), Tour Viger, 900 St-Denis St. Montreal, Quebec, Canada H2X 0A9, USA; E-mail: jolanta. gutkowska@umontreal.ca.

C 2018 Jankowski M, Gutkowska J. This article is distributed under the terms of the Creative Commons Attribution 4.0 International License.
Coronary heart disease (CHD) is the leading cause of death in the world. Ischemia in the form of acute myocardial infarction (MI) is treated by the rapid restoration of blood flow through reperfusion strategies, which often result in ischemia-reperfusion injury of the myocardium. The loss of cardiomyocyte contractile function remains the largest challenge to functional recovery of the heart from an episode of acute MI. Evidence from recent years has demonstrated that the neuropeptide hormone oxytocin (OT) has the capacity to attenuate myocardial damage resulting from ischemia-reperfusion injury in animal models ${ }^{1-4}$. As a result of these findings, new therapies in the prevention of MI-induced injury are proposed. In this context, researchers from the Sheba Medical Center in Ramat Gan, Israel, initiated a clinical trial entitled "Theimpact of oxytocin administration on short and long term prognosis in patients undergoing ST elevation myocardial infarction" (https://clinicaltrials.gov/ct2/show/ NCT03001635). In this on-going trial, patients with the diagnosis of STEMI on admission receive conventional treatment or conventional treatment plus an intravenous OT infusion for a period of 6 hours. Main outcome measures following treatment consist of infarct size, ejection fraction, and the incidence of ventricular arrhythmias.

Lethal reperfusion injury arises from the rapid normalization of physiological conditions after a period of ischemia. High intracellular concentrations of $\mathrm{Ca}^{2+}$ as a consequence of the re-establishment of ionic homeostasis and the production of reactive oxygen species (ROS) trigger the opening of the mitochondrial permeability transition pore (mPTP), a large conductance pore that forms on the mitochondrial membrane. Sustained opening of the MPTP during reperfusion collapses the mitochondrial membrane potential and releases mitochondrial proteins into the cytosol which, in turn, activates intracellular pathways that lead to cell death by apoptosis and necrosis ${ }^{5}$. During ischemia, cell death is prevented as a lower intracellular $\mathrm{pH}$ inhibits the formation of the mPTP. Approximately $50 \%$ of the final infarct size from cell death is believed to be preventable through conditioning strategies.

An attempt to explain the molecular mechanisms by which OT protects cardiomyocytes from ischemia-reperfusion injury was presented in a paper by Gonzalez-Ryes at al. titled "Molecular mechanisms underlying oxytocin-induced cardiomyocyte protection from simulated ischemia-reperfusion" ${ }^{\prime \prime}$. The study was executed using H9c2 cells initially isolated from ventricular muscle of the 
BDIX rat ${ }^{7}$. However, H9c2 cells do not display pacemaker potentials and the ability to contract and they not display proliferative capacity that is contradictory to true cardiomyocytes. Despite of these limitations, this cell line expresses cardiomyocyte signaling pathways ${ }^{8}$ and serves as an established in vitro model for the study of cardiomyocytes in cardiac pathologies, including cardiac hypertrophy ${ }^{9}$. The results of this study demonstrate that OT directly acts on $\mathrm{H} 9 \mathrm{c} 2$ cells to improve their survival during the acute phase of simulated ischemia and reperfusion ${ }^{6}$. OT treatment increases intracellular $\mathrm{Ca}^{2+}$, which corroborates the observation that an elevation in intracellular $\mathrm{Ca}^{2+}$ results in mitochondrial $\mathrm{Ca}^{2+}$ overload and ROS generation. Further findings suggest that OT-induced mitochondrial ROS production and eNOS activation may be mediated by OT receptor-linked transactivation of the upstream kinases Akt and ERK1/2. Indeed, the PI3K/Akt and ERK1/2 kinase pathways are redox sensitive, potentially enabling kinase activation by OT-induced mitochondrial ROS generation. The following eNOS phosphorylation results in nitric oxide (NO) production as reported in this study. Furthermore, we propose that OT evokes mitochondrial ROS generation in $\mathrm{H} 9 \mathrm{c} 2$ cells via a $\mathrm{Ca}^{2+}$-dependent mechanism, leading to activation of ERK1/2, Pi3K/Akt, and eNOS; with rapid release of NO. These results strongly suggest the involvement of the reperfusion injury salvage kinase (RISK) pathway which includes pro-survival kinases such as phosphoinositide 3-kinases (Pi3K)/Akt and extracellular signal-regulated kinases 1 and 2 (ERK1/2), whose signalisation is thought to converge on the activation of cGMP-dependent protein kinase (PKG) and protein kinase $\mathrm{C}$ (PKC) $\varepsilon$, causing the inhibition of prolonged mPTP opening. This cardioprotective pathway induced by OT was later disclosed in studies using the isolated perfused heart ${ }^{3,10}$ as well as in the ischemic, diabetic $\mathrm{db} / \mathrm{db}$ mouse model of type 2 diabetes ${ }^{11}$. Interestingly, from a clinical perspective, important reductions in infarct size are also observed when OT is administered before or at the onset of the reperfusion period ${ }^{1}$. In agreement with this hypothesis, pharmacological manipulation of this pathway represents a clinically applicable alternative to ischemic conditioning. Moreover, because OT increases glucose metabolism in hypoxic neonatal cardiomyocytes through a pathway independent of the insulin receptor ${ }^{12}$, this neuropeptide can potentially overcome the insulin resistance at the level of the cardiomyocyte observed during ischemia and reperfusion ${ }^{13}$. From a cardiac performance point of view, increasing glucose metabolism in the ischemic and reperfused heart attenuates $\mathrm{Ca}^{2+}$ overload, decreases cardiomyocyte cell death, and improves functional recovery ${ }^{14}$.

The evidence that OT exerts a cardioprotective role in myocardial infarction and experimental ischemiareperfusion injury are strongly supported by data. However, there were some points in our paper that were raised and stimulated discussion. Polshekan, Jamialahmadi ${ }^{10}$ have demonstrated in the isolated perfused rat heart model that OT exhibits anti-ischemic effects when it was administered at the onset of reperfusion. They suggested therefore that OT cardioprotection has predominantly postconditioning effects by activation of the RISK pathway. Although the authors have not reported data showing any potential preconditioning benefit of OT, the mechanisms of action during reperfusion in isolated cells are not the same as those in whole heart systems. We have already reported that several factors, including vasodilatation, negative inotropic and chronotropic effects are attributed to OT only in the isolated perfused hear $\mathrm{t}^{15}$. Indeed, the negative inotropic effect triggered by OT treatment was linked to myocardial protection against infarction in the study by Ondrejcakova et al. ${ }^{16}$. On the molecular level, OT treatment can modify numerous paracrine factors released by cardiomyocytes and other cardiac cells in ischemic conditions, leading to functional benefits on the heart ${ }^{17}$.

In our study using H9c2 cells, the use of a selective OTR antagonist or reduction of OTR concentration by siRNA treatment suppressed the protective effects of $\mathrm{OT}^{6}$. This is consistent with observation that in the early period after myocardial infarction in the rat, expression of OTR mRNA is significantly downregulated and then gradually increases above the normal levels following OT treatment ${ }^{1}$. This can be recognized as a beneficial effect because downregulation of the cardiac OTR has been linked with development of ischemia in diabetic and obese mouse models ${ }^{18}$. However, in a recent study, Wsol et al. ${ }^{19}$ have demonstrated that OTR mRNA expression was lower but OTR protein levels were elevated about 10 -fold in both left and right ventricles 4 weeks after acute MI in the rat. This led the authors to infer that post infarct heart failure is associated with an increased activity of the intracardiac OT system. The results of this study were substantiated by a recent study by Jung et al. ${ }^{20}$ in which a mice model of cardiac-specific overexpression of OTR exhibited compromised left ventricular function and increased mouse mortality. Although the molecular mechanisms explaining these results were not explored, the main findings of this study provide further evidence of the importance of the cardiac OT system in ischemic conditions. In examining the role of this system as being either beneficial or detrimental, it is important to consider the fine balance that exists between cardiac OTR versus the OT levels produced locally and the concentration of OT present in the circulation. Also of importance in this cardiac-specific overexpression of OTR model is the role of the argininevasopressin system (hormone and V1/V2 receptors) and its potential involvement on cardiac function ${ }^{15}$. Moreover, in addition to previously published data, the results of this study raise the important question on how naturally occurring variations in the cardiac OT system can influence pathology and physiological processing of the heart. 


\section{References}

1. Jankowski M, Bissonauth V, Gao L, et al. Anti-inflammatory effect of oxytocin in rat myocardial infarction. Basic Res Cardiol. 2010; 105(2): 205-18.

2. Alizadeh AM, Mirzabeglo P. Is oxytocin a therapeutic factor for ischemic heart disease. Peptides. 2013; 45: 66-72.

3. Ondrejcakova M, Barancik M, Bartekova M, et al. Prolonged oxytocin treatment in rats affects intracellular signaling and induces myocardial protection against infarction. General physiology and biophysics. 2012; 31(3): 261-70.

4. Kobayashi H, Yasuda S, Bao N, et al. Postinfarct treatment with oxytocin improves cardiac function and remodeling via activating cell-survival signals and angiogenesis. Journal of cardiovascular pharmacology. 2009; 54(6): 510-9.

5. Bernardi P, Di Lisa F. The mitochondrial permeability transition pore: molecular nature and role as a target in cardioprotection. Journal of molecular and cellular cardiology. 2015; 78: 100-6.

6. Gonzalez-Reyes A, Menaouar A, Yip D, et al. Molecular mechanisms underlying oxytocin-induced cardiomyocyte protection from simulated ischemia-reperfusion. Molecular and cellular endocrinology. 2015; 412: 170-81.

7. Kimes BW, Brandt BL. Properties of a clonal muscle cell line from rat heart. Exp Cell Res. 1976; 98(2): 367-81.

8. Branco AF, Pereira SP, Gonzalez S, et al. Gene Expression Profiling of H9c2 Myoblast Differentiation towards a Cardiac-Like Phenotype. PloS one. 2015; 10(6): e0129303.

9. Watkins SJ, Borthwick GM, Arthur HM. The $\mathrm{H} 9 \mathrm{C} 2$ cell line and primary neonatal cardiomyocyte cells show similar hypertrophic responses in vitro. In Vitro Cell Dev Biol Anim. 2011; 47(2): 125-31.

10. Polshekan M, Jamialahmadi K, Khori V, et al. RISK pathway is involved in oxytocin postconditioning in isolated rat heart. Peptides. 2016; 86: $55-62$.
11. Plante E, Menaouar A, Danalache BA, et al. Oxytocin treatment prevents the cardiomyopathy observed in obese diabetic male $\mathrm{db} / \mathrm{db}$ mice. Endocrinology. 2015; 156(4): 1416-28.

12. Florian M, Jankowski M, Gutkowska J. Oxytocin increases glucose uptake in neonatal rat cardiomyocytes. Endocrinology. 2010; 151(2): 482-91.

13. Opie LH, Sack MN. Metabolic plasticity and the promotion of cardiac protection in ischemia and ischemic preconditioning. Journal of molecular and cellular cardiology. 2002; 34(9): 1077-89.

14. Karwi QG, Uddin GM, Ho KL, et al. Loss of Metabolic Flexibility in the Failing Heart. Frontiers in cardiovascular medicine. 2018; 5: 68.

15. Gutkowska J,Jankowski M, Antunes-Rodrigues J.The role of oxytocin in cardiovascular regulation. Brazilian journal of medical and biological research = Revista brasileira de pesquisas medicas e biologicas / Sociedade Brasileira de Biofisica [et al]. 2014; 47(3): 206-14.

16. Ondrejcakova $\mathrm{M}$, Ravingerova $\mathrm{T}$, Bakos J, et al. Oxytocin exerts protective effects on in vitro myocardial injury induced by ischemia and reperfusion. Can J Physiol Pharmacol. 2009; 87(2): 137-42.

17. Noiseux N, Borie M, Desnoyers A, et al. Preconditioning of stem cells by oxytocin to improve their therapeutic potential. Endocrinology. 2012; 153(11): 5361-72.

18. Gutkowska J, Broderick TL, Bogdan D, et al. Downregulation of oxytocin and natriuretic peptides in diabetes: possible implications in cardiomyopathy. The Journal of physiology. 2009; 587(Pt 19): 472536.

19. Wsol A, Kasarello K, Kuch M, Gala K, Cudnoch-Jedrzejewska A. Increased Activity of the Intracardiac Oxytocinergic System in the Development of Postinfarction Heart Failure. BioMed research international. 2016;2016:3652068.

20. Jung C, Wernly B, Bjursell M, et al. Cardiac-Specific Overexpression of Oxytocin Receptor Leads to Cardiomyopathy in Mice. Journal of cardiac failure. 2018; 24(7): 470-8. 